\title{
Verhalten der isomeren Bibromcampher gegen Salpetersäure.
}

\author{
Von J. Kachler und F. V. Spitzer. \\ (Aus dem Universitäts-Laboratorium des Prof. A. Li eben.)
}

(Vorgelegt in der Sitzung am 21. Juni 1883.)

Die isomeren Verbindungen $\mathrm{C}_{10} \mathrm{H}_{14} \mathrm{Br}_{2} 0$, der $\alpha$-Bibromcampher (Schmelzpunkt $61^{\circ}$ C.) und der $\beta$-Bibromeampher (Schmelzpunkt $115^{\circ}$ C.) werden, wie wir bereits gezeigt haben, ${ }^{1}$ durch eine geeignete Behandlung mit Natriumamalgam in sauerer Lösung in Campher zurückverwandelt und hiedurch als Campherderivate charakterisirt. In ihrem Verhalten gegen andere Reagentien erwiesen sich diese Körper jedoch vollständig verschieden.

In der erwähnten Abhandlung war es uns blos darum zu thun, Anhaltspunkte zur Charakterisirung dieser Verbindungen zu geben; zu diesem Zwecke wurden daher die meisten Reactionen nur kurz erwähnt.

Im Folgenden soll das Verhalten des $\alpha$ - und $\beta$-Bibromcamphers gegen Salpetersäure eingehender beschrieben werden.

\section{Einwirkung von Salpetersäure auf $\alpha$-Bibromcampher.}

Wenn $\alpha$-Bibromeampher in Partien von etwa $20 \mathrm{Grm}$. mit dem vierfachen Gewichte eines Gemisches aus gleichen Theilen rauchender und gewöhnlicher concentrirter Salpetersäure zusammengebracht wird, so löst sich derselbe auf und färbt die Salpetersäure dunkelbraun. Erwärmt man gelinde auf dem Wasserbade, so tritt alsbald eine stürmische Reaction ein; das Gemenge entwickelt unter starkem Schäumen massenhaft rothe Dämpfe von Stickoxyden, freiem Brom, Nitrosylbromtur neben

1 Sitzb d. k. Akad. d. Wissensch. II. Abth., Bd. 85, p. 596; Monatshefte f. Chem. 1882, p. 205. 
Bromwasserstoff und Kohlensäure. ${ }^{1}$ Diese heftige Einwirkung lässt jedoch bald nach; erhitzt man dann auf dem Sandbade, um das Ganze im Kochen zu erhalten, so bemerkt man, dass neben Salpetersäure dunkelgefärbte Tropfen in die Vorlage übergehen, die sich als schwerere Schichte abscheiden. Dabei entweichen noch immer gelbrothe, gasförmige Producte, die Stickoxyde, Bromwasserstoff und Kohlensäure enthalten.

Die Destillation wurde unter öfterem Zuriickgiessen der über der dunklen Flüssigkeitsschichte in der Vorlage angesammelten Salpetersäure so lange fortgesetzt, bis keine öltropfen mehr ubergiengen. Auf diese Weise wurden $100 \mathrm{Grm}$. $\alpha$-Bibromcampher in fiunf Operationen verarbeitet und dabei nachstehende Producte erhalten.

A) Der mit etwas Salpetersäure zurïckgebliebene Destillationsrückstand bestand aus einer rothgelben Flüssigkeit von dicker Consistenz, welche sich beim Verdünnen mit Wasser trïbt. Um die vorhandene Salpetersäure so viel als möglich zu entfernen, wurde mehrmals mit Wasser abgedampft; dabei hinterblieb zuletzt ein schwach gelblich gefärbter Syrup ( $65 \mathrm{Grm}$.), der mit heissem Wasser durch längere Zeit behandelt, sieh nur zum Theil löst, während eine dickflüssige Masse (24 Grm.) am Boden des Gefässes abgeschieden wird. Dieselbe ist in Alkohol, Äther etc. leicht löslich, zersetzt sich beim Erhitzen unter Entwicklung von Bromwasserstoff und gibt beim Kochen mit alkoholischem Kali viel Bromkalium neben einer braunen, zähen Substanz.

Wird jedoch dieser in Wasser unlösliche Theil des Destillationsrückstandes mit etwas absolutem Alkohol aufgenommen und die Lösung längere Zeit einer langsamen Verdunstung überlassen, so bilden sich am Boden des Gefässes farblose, nadelförmige Krystalle, die zu Büscheln vereinigt und zum Theil gut ausgebildet waren.

Dieselben wurden so gut wie möglich durch Absaugen von der zähen Mutterlauge getrennt und dann aus verdünntem Alkohol

1 Da Kantschuk- und Korkverbindungen zerstört werden, ist es zweckmässig, die Operation in einem Destillationsapparate vorzunehmen, der aus einem hohen Glaskolben besteht, welcher einen aufgeschliffenen Helm mit Kühler trägt. 
umkrystallisirt. Dabei haben wir blos etwa $2 \mathrm{Grm}$. vollständig weisser Krystalle erhalten, die bei $87-88^{\circ} \mathrm{C}$. schmelzen, bromund stickstoffhältig sind, beim raschen Erhitzen verpuffen und sich in Alkalien nicht lösen.

I. $0 \cdot 2596 \mathrm{Grm}$. gaben $0 \cdot 4185 \mathrm{Grm} . \mathrm{CO}_{2}$ and $0 \cdot 1250 \mathrm{Grm}$. $\mathrm{H}_{2} \mathrm{O}$

II. $0.3570 \mathrm{Grm}$. gaben $0.5760 \mathrm{Grm} . \mathrm{CO}_{2}$ und $0 \cdot 1720 \mathrm{Grm}$. $\mathrm{H}_{2} \mathrm{O}$;

III. 0.2552 Grm. gaben $0.0740 \mathrm{Grm}$. AgBr. (mit Ätrkalk);

IV. $0.3000 " \# 0.0902, "$ (nach Carius);

V. $0.4165 " \quad \Rightarrow 32.8$ C.C.N bei $17^{\circ} \mathrm{C}$ und $755.9 \mathrm{Mm}$. Druck.

Daraus berechnet sich:

I. II. III. IV. V.

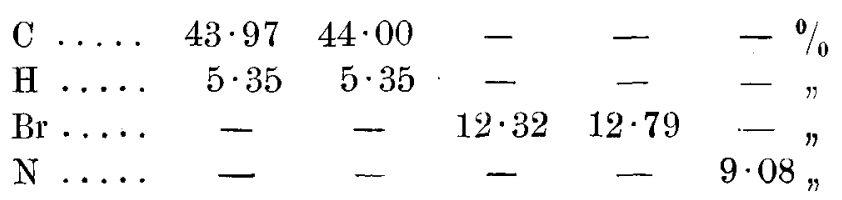

Diese Zahlen entsprechen am besten der Formel $\mathrm{C}_{24} \mathrm{H}_{33} \mathrm{Br}_{4} \mathrm{~N}_{42}$, welche verlangt:

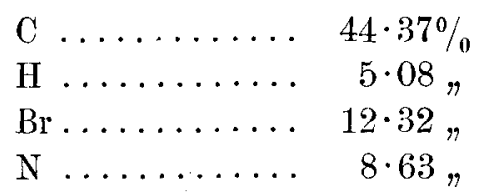

Nach dieser Zusammensetzung wäre die Substanz kein einfaches Campherderivat; es ist auch nicht ausgeschlossen, dass dieselbe ein Gemenge sei. Bei dem unzureichenden Materiale mussten wir jedoch einstweilen auf die nähere Untersuchung dieses Zwischenproductes verzichten.

Der in Wasser lösliche Theil des Destillationsriuckstandes bildete nach dem Abdampfen einen hellgelben, dicken, stark samren Syrup, der selbst nach längerer Zeit keine Krystalle abschied.

Es wurden nun Versuche gemacht, etwa darin vorhandene Säuren zu isoliren. Die wässerige Lösung mit Ätzbaryt abgesättigt 
gab keine Fällung; dagegen mit einem Überschuss des letzteren gekocht, entstand ein gelblicher, körniger Niederschlag, der abfiltrirt und mit heissem Wasser gewaschen wurde. Aus demselben konnte nach dem Zersetzen mit Schwefelsäure und Aus-

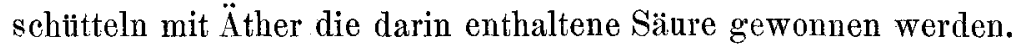
Der nach dem Abdunsten des Äthers zurückgebliebene Rückstand wurde in wenig heissem Wasser gelöst and mit Thierkohle entfärbt. Die farblose Lösung im Vacuum ïber Schwefelsäure abgedampft, schied weisse, kuglige Krystalldrusen ab, die zwischen $125-127^{\circ} \mathrm{C}$. allmählig schmolzen und im uibrigen Verbalten mit den Eigenschaften der Camphoronsäure ${ }^{1} \mathrm{C}_{9} \mathrm{H}_{12} \mathrm{O}_{5}, \mathrm{H}_{2} \mathrm{O}$ übereinstimmen. Ein Theil der Säure in wässeriger Lösung mit überschüssigem Ammon und Chlorbaryum gekocht, gab den charakteristischen Niederschlag von camphoronsauren Baryt.

$0.468 \%$ Grm. bei $180^{\circ} \mathrm{C}$. getrocknetes Baryumsalz lieferten $0 \cdot 3847$ Grm. $\mathrm{BaSO}_{4}$.

$$
\underbrace{\mathrm{Ca}_{9} \mathrm{H}_{9} \mathrm{~b} \mathrm{a}_{3} \mathrm{O}_{5}, \mathrm{H}_{2} \mathrm{O}}_{48 \cdot 87} \quad \underbrace{\text { Gefunden }}_{48 \cdot 31^{\%}}
$$

Die vom camphoronsauren Baryt abfiltrirte Lösung wurde zunächst durch Einleiten von Kohlensäure vom überschüssigen Ätzbaryt befreit, die noch voriandenen Säuren hierauf mit Bleiessig unter Vermeidung eines Überschusses gefällt, der entstandene weisse Niederschlag durch Decantation mit Wasser gewaschen und schliesslich unter heissem Wasser mit Schwefelwasserstoff zerlegt. Die vom Schwefelblei abfiltrirte Lösung, ziemlich weit eingedampft, mit Ammoniak neutralisirt, gab beim Kochen mit essigsaurem Kupfer einen voluminösen grünlichen Niederschlag, der ebenfalls abfiltrirt, mit heissem Wasser gewaschen und dann durch Schwefelwasserstoff zerlegt wurde.

Um ein klares Filtrat zu erhalten, ist es zweckmässig, die Lösung sammt dem Schwefelkupfer auf dem Wasserbade einzudampfen und erst dann zu filtriren. Die so erhaltene Lösung zur Krystallisation durch Abdampfen concentrirt, lieferte zu Drusen

1 Kachler, sitzb. d. k. Akad. đ. Wissensch. II. Abth., Juliheft 1871. Annal. d. Chem. u. Pharm. 159, p. 281. 
vereinigte Nadeln und Blättchen, die bei $160^{\circ}$ C. schmolzen. Sie erwiesen sich als nicht ganz reine Hydrooxycamphoronsäure ${ }^{1} \mathrm{C}_{9} \mathrm{H}_{14} \mathrm{O}_{6}$ (Schmelzpunkt $164.5^{\circ} \mathrm{C}$.), die als solche durch Überführung in das charakteristische bei $170^{\circ} \mathrm{C}$. schmelzende saure Ammonsalz $\mathrm{C}_{9} \mathrm{H}_{13}\left(\mathrm{NH}_{4}\right) \mathrm{O}_{6}$ erkannt werden konnte.

$0.1723 \mathrm{Grm}$. gaben $0.3214 \mathrm{Grm} . \mathrm{CO}_{2}$ und $0.0952 \mathrm{Grm}$. $\mathrm{H}_{2} \mathrm{O}$.

$$
\begin{aligned}
& \mathrm{C}_{9} \mathrm{H}_{14} \mathrm{O}_{6} \quad \text { Gefunden } \\
& \text { C } \ldots \ldots 49.54 \quad 50 \cdot 87 \% \\
& \mathrm{H} \ldots \ldots 6 \cdot 626 \cdot 14 \text {, }
\end{aligned}
$$

Die vom hydrooxycamphoronsauren Kupfer abfiltrirte Lösung gibt beim Versetzen mit Bleiessig einen gelblichen Niederschlag, aus dem nach dem Zersetzen mit Schwefelwasserstoff noch eine braune, nicht leicht krystallisationsfähige Masse von sauren Eigenschaften erhalten wurde, aus welcher sich aber nach langem Stehen ebenfalls krystallinische Hydrooxycamphoronsäure ausschied.

Der in Wasser lösliche Theil der nicht fluchtigen Producte bei der Einwirkung von Salpetersäure auf $\alpha$-Bibromcampher, besteht demnach hauptsächlich aus Camphoronsäure neben Hydrooxycamphoronsäure und geringen Mengen einer nicht krystallinisehen Substanz.

B) Der flüchtige Theil der Produete, welcher sich bei der Einwirkung von Salpetersäure auf $\alpha$-Bibromeampher in der Vorlage angesammelt hat, besteht, wie Eingangs schon erwähnt, aus zwei Schichten. Die obere branngefärbte ist Salpetersäure, welche Stickoxyde neben Bromwasserstoff gelöst enthält; die untere schwerere, beinahe schwarze Flissigkeit entwickelt an der Luft erstickende Dämpfe von Nitrosylbromïr.

Beim Vermischen mit Wasser wird das Letztere unter heftiger Reaction zersetzt, wobei eine dunkelbraun gefärbte schwere Schichte zurïckbleibt, welche behufs weiterer Reinigung mit Wasserdampf destillirt wurde.

1 Kachler, Sitzb. d. k. Akad. d.Wissensch. II. Abth., 1877. Annal. d. Chem. n. Pharm. 191, p. 143. 
Dabei konnte beobachtet werden, dass noch vor Beginn der Destillation im Kühlrohre lange nadelförmige Krystalle entstanden, welche jedoch von den ersten mit dem Wasserdampfe übergehenden öltropfen gelöst wurden.

Das darauf mit Wasser gewaschene und schliesslich mit salpetersaurem Kalk getrocknete Destillationsproduct (20 Grm.) bildet eine wasserhelle, leicht bewegliche schwere Flissigkeit, von stechendem Geruche, die sich am Lichte ziemlich rasch gelb färbt und in einer Kältemischung krystallinisch erstarrt. Beim Erwärmen ist selbe nicht ohne Zersetzung fluchtig. Mit concentrirtem Ätzkali oder mit alkoholischem Kali entsteht unter Elhitzung eine gelbe krystallinische Ausscheidung einer Kaliumverbindung.

Bei der Analyse wurden folgende Resultate erhalten:

I. 0.3962 Grm. gaben $0.0856 \mathrm{Grm}$. $\mathrm{CO}_{2}$ und $0.0130 \mathrm{Grm}$. $\mathrm{H}_{2} \mathrm{O}$

II. $0 \cdot 3220 \mathrm{Grm}$. gaben $0 \cdot 0678 \mathrm{Grm} . \mathrm{CO}_{2}$ and $0 \cdot 0118 \mathrm{Grm}$. $\mathrm{H}_{2} \mathrm{O}$;

III. $0 \cdot 3844$ Grm. gaben $0 \cdot 5243 \mathrm{Grm}$. Ag Br;

IV. $0.1215 " \quad " 13 \cdot 4 \mathrm{CCN}$ bei $16^{\circ} \mathrm{C}$. und $748 \cdot 7 \mathrm{Mm}$. Druck.

Daraus berechnen sich folgende Zahlen:

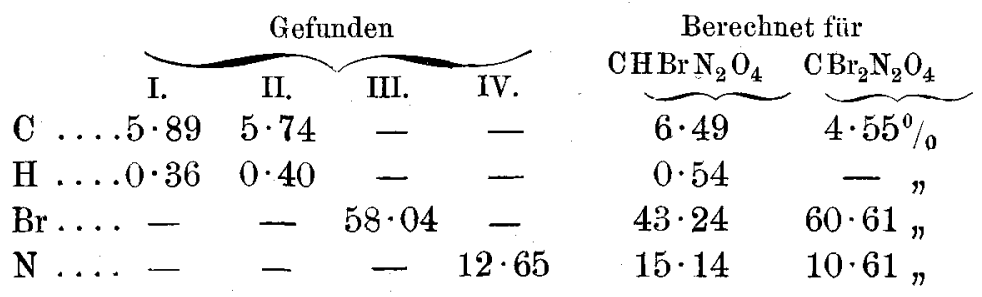

Nach diesem Ergebnisse ist die erhaltene Substanz ein Gemenge.

Um womöglich eine Trennung zu bewerkstelligen, wurde ein Theil im luftrerdünnten Raume zu destilliren versucht. Bei einem Drucke von ca. $100 \mathrm{Mm}$. fängt die Flùssigkeit an unter Braunfärbung gegen $100^{\circ} \mathrm{C}$. heftig zu sieden, dabei aber rothe Dämpfe abzugeben, wodurch das Destillat ebenfalls rothbraun gefärbt erseheint. Eine mit diesem Letzteren vorgenommene Brom- 
bestimmung ergab $58 \cdot 02 \% \mathrm{Br}$, also denselben Gehalt, wie vor der Destillation. Aus diesem Umstande mussten wir schliessen, dass der bei der Destillation mit. Wasserdampf beobachtete krystallinische Körper möglicherweise die Verunreinigung bildet und den höheren Bromgehalt bedingt.

Da, wie bereits erwähnt, aus der fraglichen Substanz mit Ätzkali eine krystallinische Kaliumverbindung entsteht, so versuchten wir solche im reinen Zustande darzustellen, um dann daraus durch Zerlegung mit einer Säure, eine einheitliche Verbindung zu gewinnen. Zu diesem Behufe wurden ca. $100 \mathrm{Grm}$. des auf die früher angegebene Weise bereiteten flüchtigen Öles in absolutem Alkohol gelöst und untel Eiskühlung alkoholisches Kali eingetragen. Sofort entstand eine reichliche Ausscheidung des gelbgefärbten krystallinischen Salzes, welche sich gut absetzte. Nachdem durch weiteren Zusatz von alkoholischem Kali kein gelber Niederschlag mehr entstand, wurde nach einigem Stehen das gebildete Kaliumsalz abfiltrirt und mit absolutem Alkohol gewaschen. Der ablaufende Alkohol ist gelb gefärbt; wird derselbe destillirt, so krystallisirt aus dem Destillationsrïckstande noch ein Theil des Kaliumsalzes neben Bromkalium aus. Die davon abfiltrirte letzte alkoholische Lange mit Wasser verdtinnt, scheidet eine schwere Flüssigkeitsschichte $a b$, in der sich nach einiger Zeit derbe, nahezu farblose Krystalle bilden, die nach dem Pressen zwischen Papier, durch Sublimation gereinigt, den Schmelzpunkt von $93^{\circ} \mathrm{C}$. zeigten. Der iberdestillirte Alkohol ist farblos und besitzt einen eigenthümlichen, an Aldehyd erinnernden Geruch; beim Vermengen mit einer grossen Menge Wasser entsteht eine Trübung und nach einiger Zeit erfolgt die Ausscheidung. von weissen nadelförmigen Krystallen (ca. $1 \mathrm{Grm}$.). Dieselben wurden auf einem Filter gesammelt und mit Wasser gewaschen, zwischen Papier gepresst, hierauf sublimirt und schmolzen dann ebenfalls bei $93^{\circ} \mathrm{C}$. Diese Substanz ist mit der eben beschriebenen, im Destillationsrückstande gefundenen, äusserst leicht sublimationsfähigen Verbindung identisch und erwies sich als Tetrabrómkohlenst off.

$0 \cdot 1664 \mathrm{Grm}$. gaben $0 \cdot 3742 \mathrm{Grm}$. AgBr.

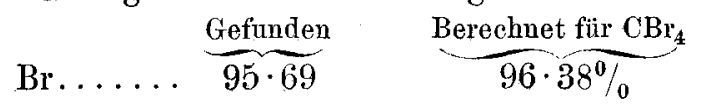


Verhalten der isomeren Bibromeampher gegen Salpetersäure. 561

Das gewonnene Kaliumsalz ist in Alkohol oder Äther nicht löslich, dagegen löst es sich im Wasser mit intensiv gelber Farbe. Aus der mit kochendem Wasser bereiteten Lösung erscheinen beim Abkühlen gelbe, schön ausgebildete, glänzende Krystalle, während beim Verdunsten der Mutterlauge neben der gelben Kaliumverbindung noch farblose Wiurfel von Bromkalinm in ziemlicher Menge erhalten werden konnten. Nur die ersten Krystallisationen gaben in wässeriger Lösung mit Silbernitrat selbst beim Kochen keine Fällung von Bromsilber, erwiesen sich somit frei von Bromkalium. Beim raschen Erhitzen verpufft die Kaliumverbindung sehr lebhaft; mit verdünnten Säuren zusammengebracht, verschwindet die gelbe Farbe und ein schweres, farbloses Öl wird abgeschieden.

Über die krystallographischen Verhältnisse dieser Kaliumverbindung hatte Herr Oberbergrath Prof. v. Zepharovich die Guite, uns Folgendes mitzutheilen:

$$
\begin{aligned}
& \text { Krystallsystem : asymmetriseh }, \\
& a: b: c=0 \cdot 7845: 1: 0 \cdot 6619 \\
(b c)= & \alpha=77^{\circ} 15^{\prime} ; \text { oP }: \infty \mathrm{P} \infty=80^{\circ} 8^{\prime} \\
(a c)= & \beta=117^{\circ} \quad 4^{\prime} ; \text { oP }: \infty \mathrm{P} \infty=115^{\circ} 54^{\prime}{ }^{\prime} \\
(a b)= & \gamma=98^{\circ} 49^{\prime} ; \infty \mathrm{P} \infty: \infty \mathrm{P} \check{\infty}=93^{\circ} 30^{\prime} .
\end{aligned}
$$

Die angegebenen Winkel liegen im ersten Octanten. Bei wechselnden Habitus wurden beobachtet:

$$
\text { oP; } \infty \mathrm{P}^{\mathrm{P}} ; \infty{ }^{\mathrm{P}} ; \infty \mathrm{P}^{\infty} ;, \mathrm{P}, \bar{\infty} . \mathrm{P}, ;{ }_{1 / 2} \mathrm{P}, ;, \mathrm{P} ; \mathrm{P} . "
$$

Die ausgefiuhrten Analysen ergaben folgendes:

I. $0.4691 \mathrm{Grm}$. gaben $0.0960 \mathrm{Grm} . \mathrm{CO}_{2}$ und $0.0137 \mathrm{Grm}$. $\mathrm{H}_{2} \mathrm{O}$

II. $0.5779 \mathrm{Grm}$. gaben $0 \cdot 1235 \mathrm{Grm}$. $\mathrm{CO}_{2}$ und $0 \cdot 0266 \mathrm{Grm}$. $\mathrm{H}_{2} \mathrm{O}$;

III. $0 \cdot 4426$ Grm. gaben $0 \cdot 1753 \mathrm{Grm} . \mathrm{K}_{2} \mathrm{SO}_{4}$;

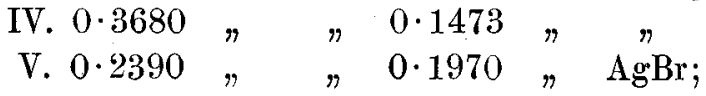

VI. $0.3680, n \quad 0 \cdot 3027, " n$

VII. $0.2978 "$ " $0 \cdot 2495 ", "$

VIII. $0 \cdot 2560 " \quad n \quad 28 \cdot 6 \mathrm{C}$. C. $\mathrm{N}$ bei $20^{\circ} \mathrm{C}$. und $747 \cdot 3 \mathrm{Mm}$.

Druck. 


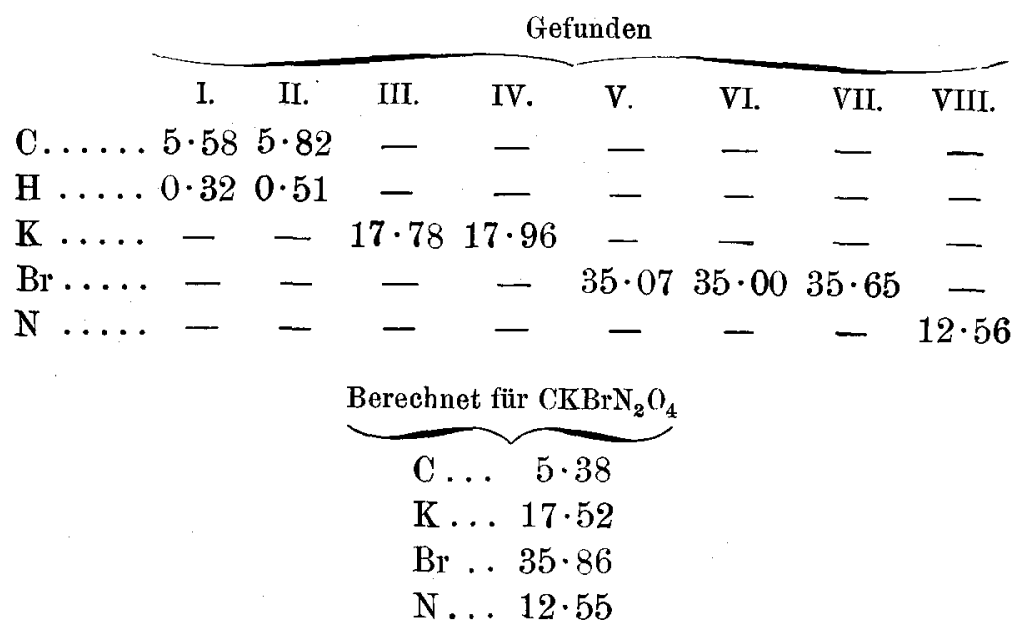

Die Kaliumverbindung ist somit Monobromdinitromethankalium. Da dieselbe aus der durch Einwirkung von Salpetersäure auf $\alpha$-Bibromcampher gewonnenen Bromnitroverbindung leicht dargestellt werden kann, halten wir es für wahrscheinlich, dass diese Letztere hauptsächlich aus Monobromdinitromethan $\mathrm{CHBr}\left(\mathrm{NO}_{2}\right)_{2}$ besteht und dass demselben Bromkohlenstoff, den wir auf die angegebene Weise isoliren konnten, beigemengt ist. Dadurch erklärt sich auch, dass die bei der Analyse gefundenen Zahlen, mit den für die Verbindung $\mathrm{CHBr}\left(\mathrm{NO}_{2}\right)_{2}$ berechneten, nicht übereinstimmen.

Wir glaubten nun durch Zerlegung des Kaliumsalzes die reine Verbindung erhalten zu können. Es wurde desshalb eine wässrige Lösung der Kaliumverbindung mit verdünnter Schwefelsäure versetzt, wobei sich eine schwere Flüssigkeitsschichte abschied und auch eine geringe Gasentwicklung auftrat. Das von der wässerigen Lösung getrennte Zerlegungsproduct wurde mit Wasser destillirt, hierauf einigemal gewaschen und mit salpetersaurem Kalk getrocknet.

I. $0 \cdot 718$ Grm. gaben $0 \cdot 1413$ Grm. $\mathrm{CO}_{2}$ und $0 \cdot 0206 \mathrm{Grm}$. $\mathrm{H}_{2} \mathrm{O}$;

II. $0 \cdot 5709 \mathrm{Grm}$. gaben $0 \cdot 1090 \mathrm{Grm} . \mathrm{CO}_{2}$ und $0 \cdot 0160 \mathrm{Grm}$. $\mathrm{H}_{2} \mathrm{O}$;

III. $0 \cdot 2947 \mathrm{Grm}$. gaben $0 \cdot 3766 \mathrm{Grm}$. $\mathrm{AgBr}$. 


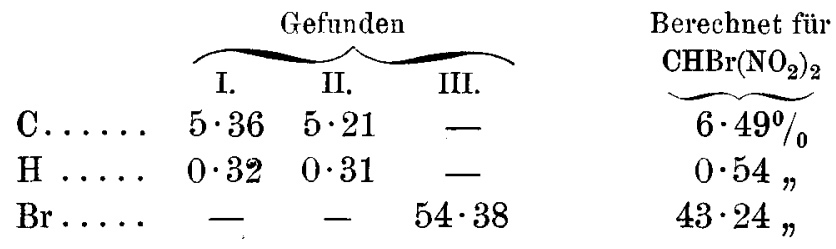

Aus diesen analytischen Daten ergibt sich, dass die aus der Verbindung $\mathrm{CKBr}\left(\mathrm{NO}_{2}\right)_{2}$ mittelst Schwefelsäure gewonnene Substanz nicht reines Monobromdinitromethan ist, sondern dass dasselbe eine bromreichere Verbindung enthält, welche sich erst bei der Zerlegung des reinen Kaliumsalzes gebildet hat. Wir haben auch thatsächlich in diesem Producte wieder Bromkohlenstoff nachgewiesen. Wird nämlich dasselbe in einem mit Kühlrohr versehenen Kölbchen einige Zeit auf dem Wasserbade gelinde erwärmt, so beschlagen sich die gektihlten Stellen bald mit langen, farblosen, strahlenförmig auslaufenden Krystallen, die als Tetrabromkohlenstoff erkannt wurden.

Dieser Gehalt an Bromkohlenstoff mag auch die Ursache sein, dass sowohl die ursprüngliche aus $\alpha$-Bibromcampher und Salpetersäure erhaltene, als auch die aus dem reinen Kaliumsalze durch Säuren abgeschiedene Substanz bei der Behandlung mit alkoholischem Kali eine mit Bromkalium gemengte Kaliumverbindung liefert.

Es lag die Vermuthung nahe, dass das Monobromdinitromethan selbst eine Zersetzung unter Bildung von Tetrabromkohlenstoff erleidet, da die bekannten Chlornitromethanverbindungen ein ähnliches Verhalten zeigen. Wir versuchten desshalb die Zerlegung des Kaliumsalzes bei Vermeidung jeder Temperaturerhöhung in der Weise vorzunehmen, dass dabei die Anwendung eines Säureüberschusses vermieden und die freiwerdende Substanz jeder weiteren Umset/ung, sei es mit einem Theile nicht zerlegter Kaliumverbindung oder zugefügter Säure, womöglich entzogen werde. $\mathrm{Zu}$ diesem Zwecke wurden $5 \mathrm{Grm}$. des reinen Kaliumsalzes in Wasser gelöst, dann Äther zugegeben und unter Kühlung mit Wasser und fortwährendem Sehütteln die zur Umsetzung nöthige, berechnete Menge titrirter Schwefelsäure aus einem Tropftrichter allmählig zugefügt. Dabei trat mit dem letzten Tropfen der zugesetzten Säure vollständige Entfärbung 
der früher gelben, wässerigen Schichte ein. Nachdem die ätherische Lösung abgezogen, mit Wasser gewaschen, hierauf mit Chlorcalcium getrocknet war, wurde der Äther durch gelindes Erhitzen auf dem Wasserbade abdestillirt. Sobald dies aber geschehen war, trat bei gleichzeitiger Einwirkung von directem Sonnenlicht eine heftige Zersetzung der zurïckgebliebenen Substanz unter Entwicklung von rothen Dämpfen ein. Beim weiteren Erhitzen auf dem Wasserbade sammelten sich im Kuhlrohre auch wieder Krystalle von Tetrabromkohlenstoff an.

Wir haben daher neuerdings 5 Grm. des reinen Kaliumsalzes anf die eben angegebene Weise zerlegt, nur dabei den Zutritt von Licht möglichst vermieden. Der Äther wurde unter einer Glocke über Paraffin bei gewöhnlicher Temperatur abgedunstet und schliesslich im Vacuum entfernt. Es blieb dabei eine nahezu farblose, etwas trübe Flüssigkeit zurïck, die nach vollständiger Klärung eine geringe Menge Wasser an der Oberfläche absehied, das durch sorgfältiges Abpipettiren entfernt werden konnte. Das so gewonnene Product nahm, trotzdem es immer vor Zutritt des Lichtes nach Möglichkeit geschützt war, ziemlich rasch eine gelbe Farbe an.

I. $0 \cdot 7391 \mathrm{Grm}$. gaben $0 \cdot 1562 \mathrm{Grm} . \mathrm{CO}_{2}$ und $0 \cdot 0284 \mathrm{Grm}$. $\mathrm{H}_{2} \mathrm{O}$

II. $0 \cdot 3560 \mathrm{Grm}$. gaben $0 \cdot 4474 \mathrm{Grm} . \mathrm{AgBr}$;

III. $0.4060 \mathrm{Grm}$. gaben $47 \cdot 5$ C. C. N bei $24^{\circ} \mathrm{C}$. und $744.7 \mathrm{Mm}$. Druck.

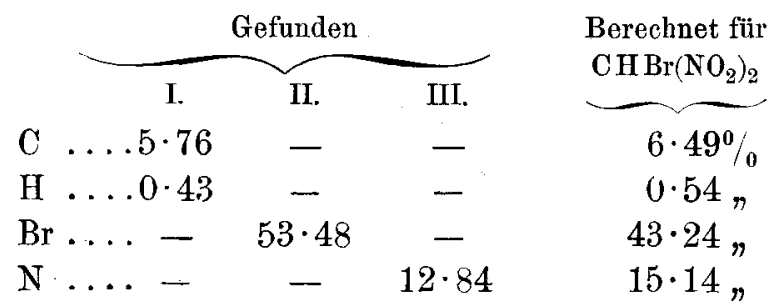

Daraus ist $z u$ ersehen, dass man trotz aller Vorsicht bei der Zerlegung der Verbindung $\mathrm{CKBr}\left(\mathrm{NO}_{2}\right)_{2}$ mittelst Säuren, nicht reines Monobromdinitromethan erhalten kann; der Grund ist wohl in der leichten Zersetzlichkeit dieser Substanz zu suchen. 
Verhalten der isomeren Bibromcampher gegen Salpetersäure. 565

Es muss hier erwähnt werden, dass Losanitseh ${ }^{1}$ durch Einwirkung von Salpetersäure auf Tribromanilin oder Äthylenbromid eine brom- und stickstoffhältige Verbindung bekommen hat, die in alkoholischer Lösung mit wässerigem Kali behandelt, ebenfalls ein gelbes krystallinisches Kaliumsalz von der Zusammensetzung $\mathrm{CK} \operatorname{Br}\left(\mathrm{NO}_{2}\right)_{2}$ lieferte, welches ohne Zweifel mit der von uns beschriebenen Kaliumverbindung identisch ist. Losanitsch behauptet nun, dass beim Zersetzen desselben mit Sätren unter Gasentwicklung Dibromdinitromethan $\mathrm{CBr}_{2}\left(\mathrm{NO}_{2}\right)_{2}$, allerdings in geringer Ausbeute entsteht; nebenbei aber auch eine weisse, krystallinische, leicht flüchtige, campherartig: riechende Verbindung gebildet werde.

Er meint, es sei leicht zu ersehen, dass hiebei mindestens zwei Moleküle des Kaliumsalzes ein Molekül Dibromdinitromethan liefern. Unsere Versuche bestätigen diese Angabe nicht, da wir niemals die Verbindung $\mathrm{CBr}_{2}\left(\mathrm{NO}_{2}\right)_{2}$ erhalten konnten. Es ist vielmehr anzunehmen, dass die von Losanitsch bemerkte krystallinische, fluchtige Substanz Tetrabromkohlenstoff gewesen sei, welcher wohl auch dem von ihm aus dem Kaliumsalze erhaltenen Producte beigemengt war. Auf diese Weise wäre es zu erklären, dass er bei der Analyse Zahlen erhalten hat, welche mit den für $\mathrm{CBr}_{2}\left(\mathrm{NO}_{2}\right)_{2}$ berechneten gut übereinstimmen.

Auf welche Weise auch die freie Bromnitroverbindung dargestellt wurde, immer konnte darin Tetrabromkohlenstoff nachgewiesen werden, dessenungeachtet war der gefundene Bromgehalt niedriger als der für $\mathrm{CBr}_{2}\left(\mathrm{NO}_{2}\right)_{2}$ berechnete.

Wir grïnden hierauf die Ansicht, dass sowohl die bei der Einwirkung von Salpetersäure auf $\propto$-Bibromeampher, als auch die aus der Verbindung $\mathrm{CKBr}\left(\mathrm{NO}_{2}\right)_{2}$ dargestellte Substanz hauptsächlich aus Monobromdinitromethan $\mathrm{CHBr}\left(\mathrm{NO}_{2}\right)_{2}$ besteht und Tetrabromkohlenstoff enthält; möglicherweise ist auch noch eine andere Verbindung in geringer Menge beigemengt.

Wir haben demnach gefunden, dass bei der Einwirkung von concentrirter Salpetersäure auf $\alpha$-Bibromeampher, Camphoron-

1 Ber. d. d. chem. Ges., XV., 1882, 471. Ber. d. d. chem. Ges., XVI., $1883,51$. 
säure, Hydrooxycamphoronsäure und Bromdinitromethan neben Kohlensäure, Bromwasserstoff und Nitrosylbromür als Hauptproducte gebildet werden.

\section{Einwirkung von Salpetersäure auf $\beta$-Bibromcampher.}

Wie wir bereits in unserer frutheren Abhandlung ${ }^{1}$ angegeben: haben, wird der $\beta$-Bibromcampher von Salpetersäure nicht leicht. angegriffen und bei der Destillation mit rauchender Salpetersäure hauptsächlich Bibrommononitrocampher $\mathrm{C}_{10} \mathrm{H}_{13} \mathrm{Br}_{2}\left(\mathrm{NO}_{2}\right) \mathrm{O}$ gebildet.

Um zu untersuchen, ob nicht neben diesem Körper noch andere Producte entstehen, haben wir diese Reaction mit grösseren Mengen ausgeführt. $50 \mathrm{Grm}$. $\beta$-Bibromcampher wurden. auf dieselbe Weise wie der $\alpha$-Bibromeampher, mit dem Gemenge von concentrirter und rauchender salpetersäure durch mehrere Tage erhitzt und die abdestillirte Säure öfters ersetzt.

Unter den flüchtigen Producten konnte neben Stickoxyden, Bromwasserstoff, Kohlensäure und geringen Mengen von Nitrosylbromür, kein schweres 01 bemerkt werden. Der Destillationsruckstand bildete nach dem Vermischen mit Wasser zwei Schichten, von denen die untere nahezu fest wurde. Die von dem festen Producte getrennte wässerige Lösung gab nach öfterem Abdampfen mit Wasser, etwa 2 Grm. einer dickflüssigen Masse, die noch Bromwasserstoff enthielt. Dieselbe wurde in ähnlicher Weise, wie der in Wasser lösliche Destillationsrückstand von der Behandlung des $\boldsymbol{\alpha}$-Bibromeamphers mit Salpetersäure, auf darin etwa enthaltene Säuren untersucht. Dabei konnte jedoch nur etwas $0 \times$ alsäure mit Sicherheit nachgewiesen werden.

Das erwähnte, in Wasser unlösliche krystallinische Product gab nach dem Umkrystallisiren ans Alkohol $30 \mathrm{Grm}$. Bibrommononitrocampher $\mathrm{C}_{10} \mathrm{H}_{13} \mathrm{Br}_{2}\left(\mathrm{NO}_{2}\right) 0$, während nur wenig einer schmierigen Mutterlauge hinterblieb.

1 In dieser Abhandlung ist bei der Angabe der Krystallform des 3-Bibromcamphers folgender Druckfehler zu corrigiren: Seite 606, Zeile 12 von oben, lies 0.5206 statt 1.05206 ; ferner ist auf Seite 612 die Formel der der Campherkohlensäure $\mathrm{C}_{22} \mathrm{H}_{32} \mathrm{O}_{6}$, statt $\mathrm{C}_{20} \mathrm{H}_{32} \mathrm{O}_{6}$ zu lesen. 
Verhalten der isomeren Bibromcampher gegen Salpetersäure. 567

Der nochmals aus einem Gemische von Äther und Alkohol umkrystallisirte Bibrommononitrocampher bildet farblose Nadeln oder Säulchen, die genan bei $130^{\circ}$ C. schmelzen, in Wasser unlöslich, dagegen in Alkohol oder Äther leicht löslich sind.

Über die krystallographischen Verhältnisse stellte uns Herr Oberbergrath Prof. v. Zepharovich folgende Daten zur Verfitgung.

$$
\begin{aligned}
& \text { „Krystallsystem: rhombisch, } \\
& a: b: c=0 \cdot 8474: 1: 0 \cdot 5684
\end{aligned}
$$

Nadeln oder Säulchen nach der Brachyaxe;

Formen: $\infty \mathrm{P} \check{\infty}, \mathrm{P} \check{\infty}, \mathrm{P} \bar{\infty}>\infty \mathrm{P} \overline{2} . " ،$

Der Bibrommononitrocampher $\mathrm{C}_{10} \mathrm{H}_{13} \mathrm{Br}_{2}\left(\mathrm{NO}_{2}\right) \mathrm{O}$ geht bei der Reduction in Amidocampher $\mathrm{C}_{10} \mathrm{H}_{15}\left(\mathrm{NH}_{2}\right) \mathrm{O}$ iber. Es wurde erstere Verbindung in einer Lösung von Eisessig mit Zinn einige Zeit am Rückflusskühler gekocht, das Product nach dem Verdïnnen mit Wasser mittelst Schwefelwasserstoff vom Zinn befreit, die Lösung mehrmals unter Zusatz von Salzsäure auf dem Wasserbade abgedampft und dann mit Wasser aufgenommen, wobei etwas unlösliche, nicht krystallinische Substanz zurückblieb. Aus der concentrirten wässrigen Lösung schieden sich in Wasser sehr leicht lösliche Krystalle ab. Das daraus gewonnene Platindoppelsalz, wurde in drei Fractionen getheilt. Die beiden ersten enthielten zwei verschieden krystallisirende Substanzen, rothgelbe, körnige Krystalle neben hellgelben sternförmig gruppirten Nadeln. In der letzten Fraction waren nur Spuren der ersten Substanz bemerkbar und bei der Analyse gaben $0 \cdot 1415$ Grm. 0.0396 Grm. Pt.

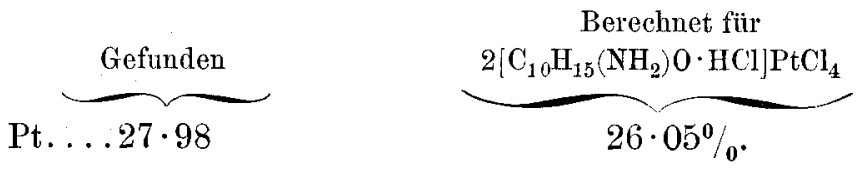

Dieses Platindoppelsalz stimmt in seinen Eigenschaften mit dem von $R$. Schiff ${ }^{1}$ aus dem salzsauren Amidocampher

1 Gaz. chim. ital. t. X, 1880; Ber. d. d. chem. Ges. XIII, 1880, 1404. 
568 Kaebler u. Spitzer. Verhatten d. isom. Bibromeampherete.

erhaltenen iberein. Der etwas zu hoch gefundene Platingehalt rtihrt von einer geringen Beimengung an Platinsalmiak her, welcher in der ersten Fraction in grösserer Menge vorhanden ist.

Wie wir dies bereits in der eingangs angeftihrten Abhandlung hervorgehoben haben, wirkt Salpetersäure auf die beiden isomeren Bibromcampher versehieden ein. Während der $\alpha$-Bibromcampher dabei leicht die aus Campher entstehenden Säuren mit 9 Kohlenstoffatomen und Bromdinitromethan liefert, wobei aber immer Producte einer vollständigen Zersetzung, wie Kohlensäure, Bromwasserstoff etc. auftreten, entsteht aus dem $\beta$-Bibromcampher zunächst dessen Nitrosubstitutionsproduct, dass dann weiter durch die oxydirende Wirkung der Salpetersäure, direct in Kohlensänre, Bromwasserstoff etc. zerfällt.

Es wird unsere weitere Aufgabe sein, die aus dem Campher entstehenden Säuren, speciell diejenigen mit 9 Kohlenstoffatomen näher zu untersuchen, um dann im Zusammenhange mit den bereits erhaltenen Resultaten, zu weiteren Anhaltspunkter bezüglich der Constitution dieser Verbindungen zu gelangen. 